\title{
Effects of Exercise Training on the Severity and Composition of Atherosclerotic Plaque in apoE-Deficient Mice
}

\author{
Nikolaos P.E. Kadoglou ${ }^{a} \quad$ Nikolaos Kostomitsopoulos $^{\mathrm{a}} \quad$ Alkistis Kapelouzou $^{\mathrm{a}}$ \\ Petros Moustardas $^{a}$ Michalis Katsimpoulas ${ }^{a}$ Athina Giagini ${ }^{a} E_{\text {Eleni Dede }}{ }^{a}$ \\ Harisios Boudoulas $^{a} \quad$ Stavros Konstantinides $^{c}$ Panayotis E. Karayannacos ${ }^{a}$ \\ Christos D. Liapis ${ }^{b}$ \\ ${ }^{a}$ Center of Experimental Surgery, Biomedical Research Foundation, Academy of Athens, and ${ }^{\text {b}}$ Department of \\ Vascular Surgery, Medical School, University of Athens, Athens, Greece; ' ${ }^{\text {Department }}$ of Cardiology and \\ Pulmonary Medicine, Georg August University of Göttingen, Göttingen, Germany
}

\section{Key Words}

Atherosclerosis $\cdot$ Exercise $\cdot$ Plaque stability $\cdot$ Matrix

metalloproteinases $\cdot$ Tissue inhibitor of metalloproteinases 1

\begin{abstract}
Aim: To investigate the effects of exercise on atherosclerotic plaque composition, the concentration of matrix metalloproteinases (MMPs) in the atherosclerotic plaque and the systemic circulation. Methods: Ninety apolipoprotein E-deficient (apoE ${ }^{-/}$) mice (45 male) were randomized to the following groups ( $n=15$ each): control male/female; sedentary male/female; exercise male/female. Mice were kept on a 16week high-fat diet. Subsequently, the control groups were sacrificed, while the rest of the animals were placed on a normal diet for 6 more weeks. During the latter period, the exercise groups were trained daily on treadmill. At the end of the study, mice were euthanized, and blood samples as well as aortic root specimens were obtained. Results: Compared to control and sedentary animals, exercise training reduced atherosclerotic plaques $(-30 \% ; p<0.01)$ and increased elastin and collagen content in both genders $(p<0.05)$. Body weight or lipid profile did not change significantly. Decreased macrophages and MMP-9 as well as increased tissue
\end{abstract}

inhibitor of metalloproteinases 1 (TIMP-1) levels were observed in the atherosclerotic plaques of the exercise-treated groups $(p<0.05)$. Plasma concentrations of MMP-9 decreased, while plasma TIMP-1 levels increased in the exercise compared to control and sedentary groups $(p<0.05)$. Conclusions: Exercise training had a favorable effect on the size and composition of the atherosclerotic plaque in $\mathrm{apoE}^{-/-}$ mice, associated with suppressed MMP activity.

Copyright $\odot 2011$ S. Karger AG, Basel

\section{Introduction}

It is well known that an inflammatory process contributes significantly to the development and progression of atherosclerosis. Studies have shown that inflammation modulates extracellular matrix (ECM), regulates vascular wall homeostasis and contributes to atherosclerotic plaque instability $[1,2]$. Although exercise training has been suggested to slow atherosclerotic plaque progression and enhance plaque stability, the precise underlying mechanisms for these beneficial effects remain to be defined [3].

Matrix metalloproteinases (MMPs), a family of zincproteolytic enzymes that degrade various components of

\section{KARGER}

Fax +41613061234 E-Mail karger@karger.ch www.karger.com

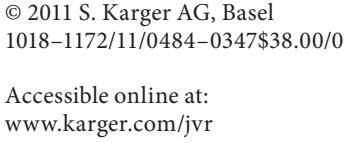

Dr. Nikolaos P.E. Kadoglou

Center of Experimental Surgery, Biomedical Research Foundation

Academy of Athens, 4 Soranou Ephessiou Str.

GR-11527 Athens (Greece)

Tel. +30 210659 7376, Fax +30 210659 7365, E-Mail nkado@ med.uoa.gr 


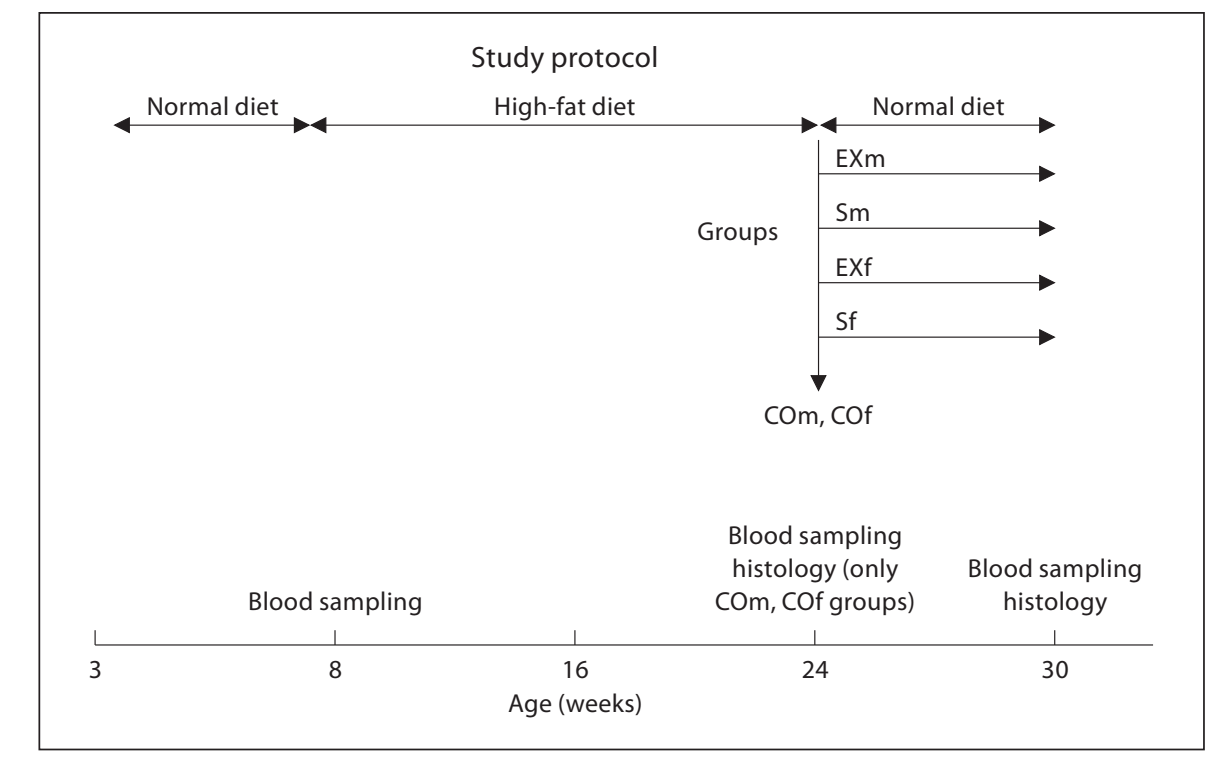

Fig. 1. Study design. $\mathrm{COm}=$ Control male; COf $=$ control female; Sm = sedentary male; $\mathrm{Sf}=$ sedentary female; EXm = exercise male; $\mathrm{EXf}=$ exercise female.

the ECM [4], are related to inflammation. Available information supports the important role of MMPs and their inhibitors (tissue inhibitors of metalloproteinases, TIMPs) in atherogenesis and atherosclerotic plaque destabilization [5]. Despite the evidence that exercise training offers anti-inflammatory effects, information related to its effect on the inflammatory indices on the atherosclerotic plaque and the peripheral circulation, such as macrophage concentration and vascular MMP expression as well as activity, is limited [6].

The purpose of the present study was to assess the effect of exercise training on the severity and composition of atherosclerotic plaques of the aorta in an experimental animal model of apolipoprotein E-deficient $\left(\mathrm{apoE}^{-/-}\right)$ mice. In addition, the effect of training on plaque and systemic MMP activity was evaluated.

\section{Methods}

Animals and Experimental Protocol

ApoE ${ }^{-/-}$mice were obtained from Charles Rivers Laboratories (Milan, Italy). Mice had been backcrossed for 10 generations to the C57BL/6 genetic background and they mated to produce F1 generation. The $\mathrm{F} 1 \mathrm{ApoE}^{-/-}$mice comprised the experimental population. A total of $90 \mathrm{ApoE}^{-1-}$ mice, male $(\mathrm{n}=45)$ and female $(\mathrm{n}=45)$, were entered into the experimental protocol at the age of 8 weeks (18-20 g; fig. 1). All animals were housed in animal rooms under specific pathogen-free conditions at a room temperature of $22 \pm 1{ }^{\circ} \mathrm{C}$, with $55 \pm 10 \%$ relative humidity, a 12/12hour light/dark cycle starting at 7:00 a.m. and with a light density of 300 Lux measured $1 \mathrm{~m}$ above the floor in the middle of the room. Animal rooms were operated with a positive air of $0.6 \mathrm{~Pa}$. Tap water in drinking bottles and vacuum-packed pelleted food were provided ad libitum.

$\mathrm{ApoE}^{-/-}$mice were weaned at 3 weeks and fed normal chow diet until 8 weeks of age, at which time mice were randomly assigned to the following groups of 15 mice each: (1) control males; (2) sedentary males; (3) exercise males; (4) control females; (5) sedentary females; (6) exercise females. Body weight and blood samples were obtained at baseline, that is, the 8 th week of age, and mice were then put on a Western-type diet (42\% of total calories from milk fat and $0.15 \%$ from cholesterol; Harlan Teklad TD 88137; Harlan, Boxmeer, The Netherlands) and maintained on this diet for 16 weeks. At the end of the 16th week of the study protocol (24th week of age), control groups were sacrificed.

Long-term administration of Western-type diet has been demonstrated to induce lesions similar to those of human fibrofatty lesions [7]. However, it is well documented that high-fat diet per se is associated with increased inflammatory milieu in animals [8]. Taken together, the remaining groups (sedentary and exercising) were switched back to normal diet (cholesterol $0.002 \mathrm{~g} / \mathrm{kg}$ of feed; total fat $45 \mathrm{~g} / \mathrm{kg}$ of feed) for 6 weeks, just to limit the confounding effects of concomitant high-fat feeding. Sedentary groups were confined to their cages, while exercise mice followed an exercise training protocol for the same time period. After dietary and/or exercise treatment, mice were euthanized following body weight measurement and aorta excision. Blood samples were obtained just before the euthanasia. Study design is presented in figure 1 . Resting heart rate was recorded using a computerized data acquisition system (DSI Systems, Tilburg, The Netherlands). For this purpose, we used an additional 4 mice for each group. Those mice were not included in statistical analysis for biochemical and histological measurements; however, they underwent the same experimental procedures (such as feeding, blood sampling, exercise training or sedentary lifestyle), in their respective groups, until euthanasia. Just before euthanasia time, we implanted minimally invasive devices, and resting heart rate was recorded for $24 \mathrm{~h}$. 
The study complied with the Guide for the Care and Use of Laboratory Animals published by the US National Institutes of Health. The protocol was approved by the Ethics Committee for Animal Experimentation of the Foundation of Biomedical Research, Academy of Athens and the competent Veterinary Service.

\section{Exercise Protocol}

Exercise training was performed on a motorized rodent treadmill with electrical shock-plate incentive (Exer-6M Open Treadmill; Columbus Instruments, Columbus, Ohio, USA) for 6 weeks, 5 days a week. A modified version of a previously evaluated exercise protocol was used [9]. During the first 2 weeks, there was a gradual increment in exercise intensity. In particular, the duration of each session increased during the first week from 15 to 45 min, with a 2 -min rest interval at the middle of the session, while the speed of the treadmill was increased from 8 to $12 \mathrm{~m} / \mathrm{min}$. In the second week, the exercise time was further increased to 60 $\mathrm{min} / \mathrm{session}$, with an intermediate 2 -min rest interval, while speed approached $15 \mathrm{~m} / \mathrm{min}$. The latter exercise parameters remained constant until the end of the 6th exercise week. The slope of the treadmill was kept unchanged at $5^{\circ}$ throughout the training period.

\section{Tissue Preparation and Quantification of Atherosclerosis}

At death, the aorta was washed thoroughly with injection of phosphate-buffered saline through the left ventricle of the heart. Subsequently, the heart and the entire aorta until the iliac bifurcation were excised en bloc and maintained in $10 \%$ buffered formalin for $24 \mathrm{~h}$. They were then embedded in paraffin and sections were obtained following the protocol of Tangirala et al. [10]. The heart was initially dissected transversely just below the level of atria and serial $7-\mu \mathrm{m}$ sections were cut from the apex towards the base of the heart until the aortic valve leaflets appeared. From this point, the aortic root was sectioned transversely into $7-\mu \mathrm{m}$-thick slices, which were collected on poly-D-lysine-coated slides. In the aortic root region, 6 sections equally separated (by $70 \mu \mathrm{m}$ ) were selected and stained with hematoxylin/eosin for quantitative morphometric analysis. Atherosclerotic plaque was defined on those consecutive cross sections, on which the hematoxylin/eosin positive-stained area occupied the lumen area, circumscribed by the internal elastic lamina. The extent of atherosclerotic lesions and the total lumen area within the internal elastic lamina (in $\mu \mathrm{m}^{2}$ ) were measured in each section using computerized image analysis software (Image Pro Plus Version 4.1; Media Cybernetics, Sarasota, Fla., USA). In each section, luminal stenosis was determined as percent lesion area per total lumen area. The average luminal narrowing was then calculated from all hematoxylin/eosin-stained sections per animal and the mean $( \pm S D)$ values from all mice per group were used for atherosclerotic lesion quantification. In addition to luminal stenosis, we calculated the ratio of intima-media thickness. The thickness of intima layer, within the internal elastic lamina, was determined and the greatest value (in $\mu \mathrm{m})$ was considered for analysis in each section. Similarly, media layer thickness, confined between internal and external elastic lamina, was also measured (in $\mu \mathrm{m}$ ) at the site of the atherosclerotic plaque. Collagen and elastin were visualized using Sirius Red and Orcein staining, respectively. All measurements were carried out independently by two operators blinded to the study groups.

\section{Immunohistochemical Analysis}

Paraffin sections of the aortic arch were stained immunohistochemically with antibodies directed against Mac-3 antigen of murine macrophages (dilution 1:50; BD Pharmingen, Franklin Lakes, N.J., USA), and $\alpha$-smooth muscle isoform of actin (dilution 1:100; Biocare Medical, LLC, Concord, Calif., USA). Moreover, MMP-2 and MMP-3 were identified with corresponding polyclonal antibodies (dilution 1:300; MBL, International Corporation, Woburn, Mass., USA). A polyclonal antibody against mouse MMP-9 was purchased from AbD Serotec (Oxford, UK). Paraffin-embedded sections were examined immunohistochemically with the following antibodies: anti-mouse monoclonal antibody against TIMP-1 (dilution 1:300; Triple Point Biologics Inc., Forest Grove, Oreg., USA) and anti-mouse polyclonal antibody against TIMP-2 (dilution 1:300; Acris Antibodies GmbH, Hiddenhausen, Germany). In particular, immunostaining procedure included sequential sections, which were stained with the aforementioned antibodies, separately one for each section. Totally, quantitative assessment of the mean immunopositive area to each antibody was calculated in 6 aortic sections per animal and it was performed on samples from 10 mice per group. Two independent investigators determined, with the aforementioned computer-assisted software, the percentage of the immunopositive-stained area within the lesions, by dividing the positive-stained area by the total atherosclerotic area. Using image analysis software, staining intensity of slides was graded from 0 to 200 . Stain intensity score lower than 20 characterized unstained slides, while overstained slides were considered when that score was higher than 180. Those upper and lower limits of staining intensity were set based on our previous laboratory experiments. Immunostaining was successful when negative controls remained unstained, while the positive controls were working. Areas with staining intensity beyond those limits $(<20$ or $>180)$ were excluded from statistical analysis. As the atherosclerotic lesions of adjacent sections are almost identical, we further selected areas of interest within the atherosclerotic lesions (shoulders, necrotic core and basement membrane). Areas of interest of adjacent sections had identical limits and therefore their surfaces were equal. We then assayed the percentage of the immunopositive-stained areas (PIPSA). Colocalization was considered when the ratio of the PIPSA derived from the same areas of interest, between two adjacent sections, was almost equal $( \pm 5 \%)$ to the ratio of PIPSA derived from the whole atherosclerotic lesions (for example, PIPSA (area of interest1) $_{\text {l }}$ $\operatorname{PIPSA}_{(\text {area of interest2) }}$ : $\operatorname{PIPSA}_{\text {(plaquel) }} / \mathrm{PIPSA}_{\text {(plaque }) \text { ) }}$.

\section{Biochemical Measurements}

At the beginning ( 8 weeks) of the study, mice were fasted overnight and blood samples (approx. $100 \mu \mathrm{l}$ ) were obtained through puncture of the retro-orbital vein. Blood sampling was repeated at the time of death ( 30 weeks) by cardiac puncture, while the mice were under isoflurane anesthesia (fig. 1). Blood samples were centrifuged at 3,500 rpm for $5 \mathrm{~min}$ and the serum was separated. Fasting plasma glucose, total cholesterol, high-density lipoprotein cholesterol and triglyceride levels were determined in fresh plasma samples from 5 mice per group using an automated enzymatic technique (Chemwell 2910; Awareness Technology Inc., Palm City, Fla., USA). Plasma levels of MMP-2 (DMP200), MMP9 (MMP900) and TIMP-1 (MTM100) were assayed using commercially available ELISA kits for mice (R\&D Systems Inc., Minneapolis, Minn., USA). For the latter parameters, the interassay 
Table 1. Body weight, lipid levels, plasma MMP-2, MMP-9 and TIMP-1 concentrations at baseline (8 weeks) and at the end (24 weeks for control groups and 30 weeks for sedentary and exercise groups) of the study in apoE $\mathrm{E}^{-/-}$mice

\begin{tabular}{|c|c|c|c|c|c|c|}
\hline Groups & $\operatorname{COm}(n=15)$ & $\operatorname{Sm}(n=16)$ & $\operatorname{EXm}(\mathrm{n}=14)$ & $\operatorname{COf}(\mathrm{n}=15)$ & $\mathrm{Sf}(\mathrm{n}=15)$ & $\operatorname{EXf}(n=15)$ \\
\hline \multicolumn{7}{|l|}{ Weight, g } \\
\hline Baseline & $22.3 \pm 2.6$ & $22.9 \pm 3$ & $22 \pm 1.2$ & $19.83 \pm 2.98$ & $20.2 \pm 2$ & $20 \pm 1.2$ \\
\hline End & $35.3 \pm 8.1^{*}$ & $36.7 \pm 4.3^{*}$ & $31.8 \pm 3.3^{*}$ & $26.5 \pm 3.83^{*}$ & $28 \pm 4.4^{*}$ & $26.6 \pm 2.6$ \\
\hline \multicolumn{7}{|l|}{$\mathrm{TC}, \mathrm{mg} / \mathrm{dl}$} \\
\hline Baseline & $441 \pm 111$ & $419 \pm 112$ & $421 \pm 99$ & $385 \pm 102$ & $398 \pm 81$ & $401 \pm 124$ \\
\hline End & $598 \pm 312$ & $602 \pm 253$ & $506 \pm 111$ & $595 \pm 88$ & $689 \pm 113$ & $540 \pm 113$ \\
\hline \multicolumn{7}{|l|}{$\mathrm{HDL}, \mathrm{mg} / \mathrm{dl}$} \\
\hline Baseline & $81 \pm 18$ & $76 \pm 12$ & $77 \pm 19$ & $70 \pm 11$ & $69 \pm 8$ & $68 \pm 12$ \\
\hline End & $85 \pm 21$ & $73 \pm 13$ & $81 \pm 11$ & $68 \pm 9$ & $69 \pm 10$ & $71 \pm 13$ \\
\hline \multicolumn{7}{|l|}{$\mathrm{TG}, \mathrm{mg} / \mathrm{dl}$} \\
\hline Baseline & $66 \pm 19$ & $65 \pm 22$ & $69 \pm 22$ & $68 \pm 28$ & $73 \pm 20$ & $76 \pm 21$ \\
\hline End & $76 \pm 32.09$ & $78 \pm 39$ & $96 \pm 21$ & $115 \pm 21$ & $119 \pm 13$ & $104 \pm 49$ \\
\hline \multicolumn{7}{|c|}{ MMP-2, ng/ml } \\
\hline Baseline & $81.52 \pm 44.18$ & $82.75 \pm 28.11$ & $84.81 \pm 23.86$ & $81.51 \pm 36.27$ & $86.86 \pm 20.55$ & $84.96 \pm 42.51$ \\
\hline End & $123.62 \pm 45.15$ & $118.2 \pm 29.15$ & $116.66 \pm 69.21$ & $145.37 \pm 36.34$ & $159.4 \pm 40.83^{*}$ & $112.25 \pm 61.04$ \\
\hline \multicolumn{7}{|c|}{ MMP-9, ng/ml } \\
\hline Baseline & $1.4 \pm 1.1$ & $1.4 \pm 1$ & $1.9 \pm 0.9$ & $1.7 \pm 0.9$ & $2 \pm 2.6$ & $1.8 \pm 0.6$ \\
\hline End & $29.5 \pm 20.9^{*}$ & $31.7 \pm 14.2^{*}$ & $13 \pm 8.8^{*,+\circ}$ & $11.5 \pm 5^{*}$ & $11.7 \pm 5.2^{*}$ & $6.8 \pm 4.4^{*,+, ~}$ \\
\hline \multicolumn{7}{|c|}{ TIMP-1, pg/ml } \\
\hline Baseline & $765.1 \pm 479.3$ & $897.89 \pm 696.1$ & $783.4 \pm 443.5$ & $744 \pm 287.1$ & $763.4 \pm 241.5$ & $846.5 \pm 233.1$ \\
\hline End & $1,434.7 \pm 945.6^{*}$ & $1,850.83 \pm 958.2^{*}$ & $3,115.5 \pm 720.6^{*,+, o}$ & $2,021.7 \pm 988.4^{*}$ & $1,888.3 \pm 850^{*}$ & $2,503.7 \pm 869.6^{*}$ \\
\hline
\end{tabular}

$\mathrm{COm}=$ Control male; $\mathrm{COf}=$ control female; $\mathrm{Sm}=$ sedentary male; $\mathrm{Sf}=$ sedentary female; EXm = exercise male; EXf = exercise female; $\mathrm{TC}=$ total cholesterol; TG $=$ triglycerides. ${ }^{*} \mathrm{p}<0.05$, within groups, ${ }^{+} \mathrm{p}<0.05$, exercise versus control groups, ${ }^{\circ} \mathrm{p}<0.05$, exercise versus sedentary groups, in the same gender.

coefficients of variance were 7.4, 7.4, 7.8 and 7.2\%, respectively, as reported by the manufacturer. The intra-assay coefficients of variants were $5.3,2,7.8$ and $4.4 \%$, respectively, according to the manufacturer. Samples were frozen and stored at $-80^{\circ} \mathrm{C}$ until analyses were performed.

\section{Statistical Analysis}

Results are expressed as means \pm SD. Student's t test was used to compare the differences between groups of the same gender and one-way ANOVA (post hoc Tukey test) for differences between all groups. Changes in body weight and serum levels of MMP-2, MMP-9 and TIMP-1 within groups were tested by paired-samples t test. A p value $<0.05$ was considered statistically significant. All calculations were performed using SPSS (Version 13.0; SPSS Inc., Chicago, Ill., USA).

\section{Results}

Body Weight, Plasma Lipids and Resting Heart Rate

Body weight significantly increased within all groups during the study. Nevertheless, the final value was not statistically different between control, sedentary and ex- ercise groups of male or female mice. Likewise, total plasma cholesterol, high-density lipoprotein cholesterol and triglyceride concentrations were not statistically different between groups of both genders at the beginning and at the end of the study (table 1). Concerning the classical exercise training effects, we observed a considerably lower resting heart rate (bpm) in the exercise male group (571 $\pm 22 \mathrm{bpm}$ ) compared to sedentary male (656 $\pm 16 \mathrm{bpm})$ and control male $(646 \pm 15 \mathrm{bpm})(\mathrm{p}<0.05)$ groups. Similar results were documented among female groups $(\mathrm{p}<$ 0.05).

\section{Quantification of Atherosclerosis, Histochemical and Immunohistochemical Analysis}

At the time of tissue harvest, the aortic root lesion area and the degree of luminal stenosis were significantly less extensive in the exercise group compared to the control $(-33.3 \%$ in male and $-26.2 \%$ in female) and sedentary $(-31.4 \%$ in male and $-23.7 \%$ in female) groups. Similar differences in intima/media ratio were observed across groups (fig. 2). Likewise, higher collagen (Sirius red stain- 

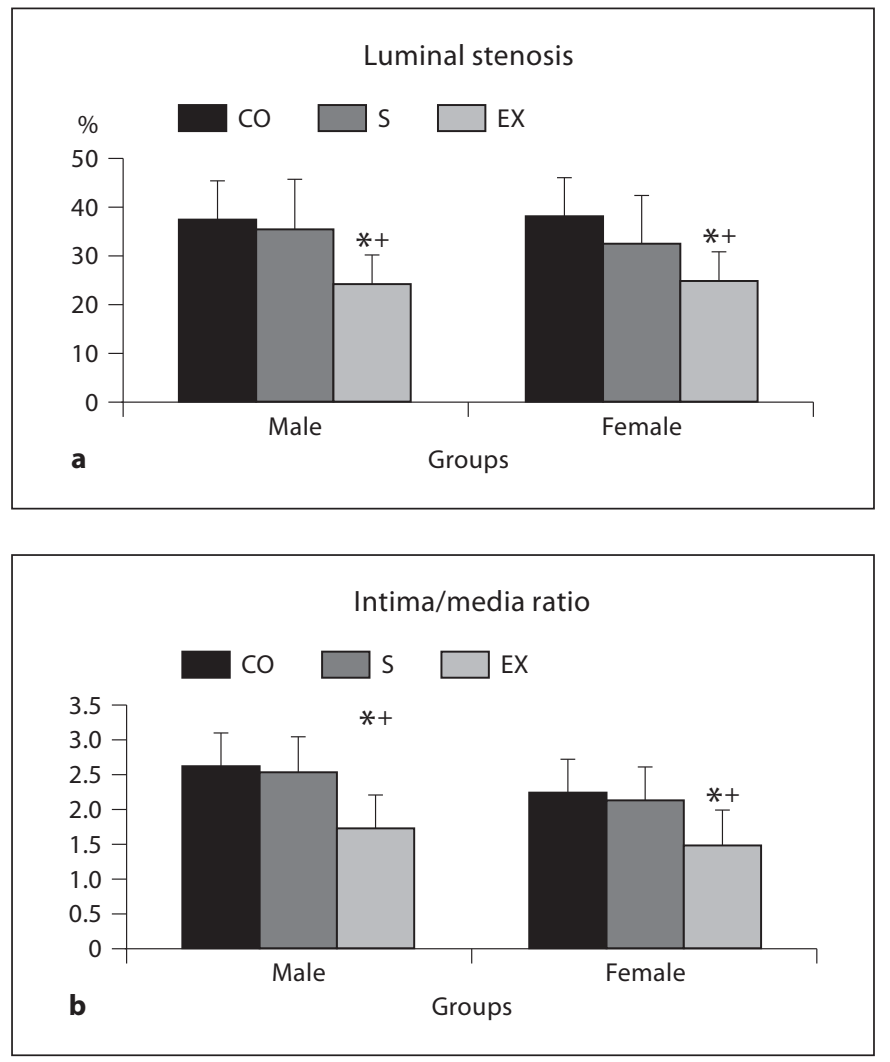

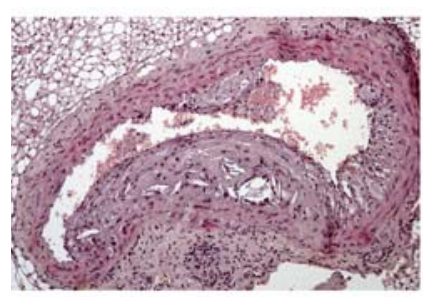

$\mathrm{COm}(\mathrm{H}-\mathrm{E})$

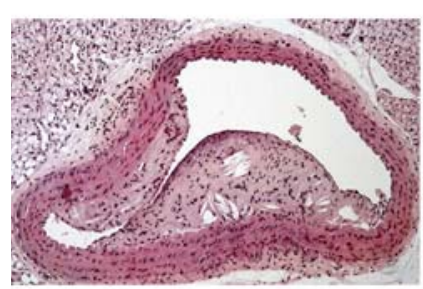

$\mathrm{Sm}(\mathrm{H}-\mathrm{E})$

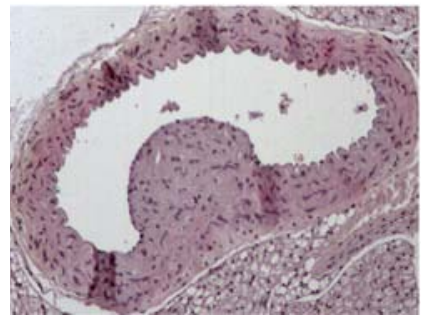

$\operatorname{EXm}(\mathrm{H}-\mathrm{E})$

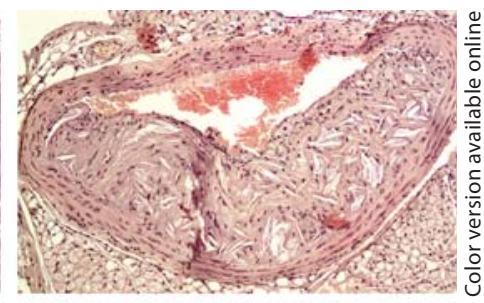

$\mathrm{COf}(\mathrm{H}-\mathrm{E})$

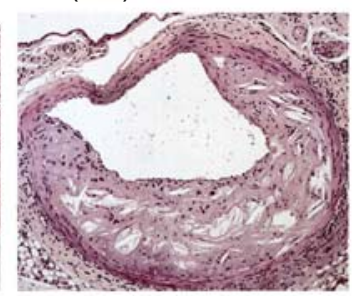

$\mathrm{Sf}(\mathrm{H}-\mathrm{E})$

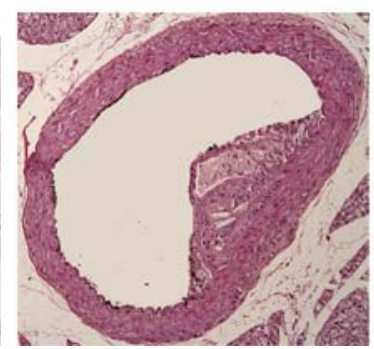

EXf (H-E)

Fig. 2. a The percentage of luminal stenosis of the aorta and the intima/media thickness were less in both male and female exercise (EX) compared to control (CO) and sedentary (S) groups; b Representative examples of the effects of exercise on plaque size using hematoxylin/eosin (H-E) staining. ${ }^{*} \mathrm{p}<0.05$, EX versus CO groups; ${ }^{+} \mathrm{p}<0.05$, EX versus $\mathrm{S}$ groups, in the same gender. $\mathrm{m}=$ Male; $\mathrm{f}=$ female.

ing) and elastin (Orcein staining) contents of atherosclerotic plaques were detected in exercise than control and sedentary groups ( $\mathrm{p}<0.05$; fig. 3,4$)$. Mice of all groups exhibited advanced lesions, consisting of a fibrous cap containing connective tissue matrix, that is, collagen and elastin fiber covering a necrotic core with foam cells (fig. 4).

Lower macrophage concentration in the atherosclerotic plaques was found in the exercise compared to untrained mice. The content of smooth muscle cells (SMCs) was slightly greater in the exercise compared to control and sedentary animals, but this difference did not reach statistically significant value (fig. 3).

The effects of exercise training on MMP and TIMP-1 expression in the lesions were also investigated. MMP-2 was equivalently detected in the atherosclerotic lesions of control, sedentary and exercise male or female groups ( $p>0.05)$. In particular, we observed positive immunostaining for MMP-2 in the area surrounding fibrous cap and co-localized with SMCs. By quantitative image analysis, a significant downregulation in MMP-9-positive area in atherosclerotic plaques was observed in the exercise male and female mice compared to their control and sedentary counterparts ( $\mathrm{p}<0.05$; fig. 3$)$. Using sequential sections, MMP-9 was mostly co-localized in macrophage areas surrounding the lipid core. MMP-9 is probably expressed in foam cells derived from macrophages and involved in remodeling of the lesions (fig. 5). Furthermore, exercise training resulted in considerable increment in TIMP-1-positive area compared to that in nonexercised groups ( $\mathrm{p}<0.01)$. The calculated MMP-9/TIMP-1 ratio was drastically less in the exercise compared to control and sedentary groups of both genders $(\mathrm{p}<0.001)$. Notably, we did not detect any difference in atherosclerotic lesion size and all histochemical and immunohistochemical parameters between control and sedentary groups $(p>0.05)$. 


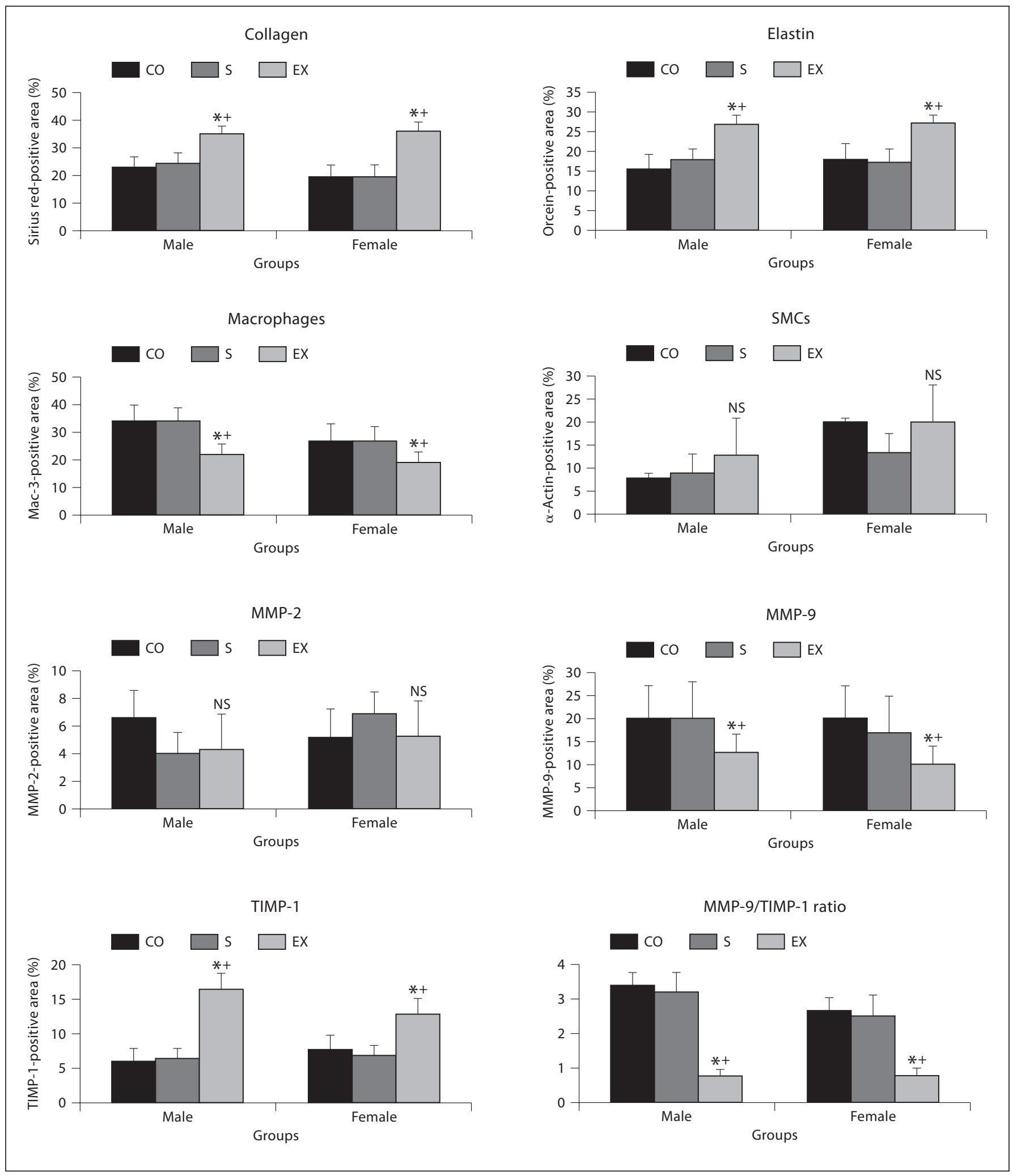

Fig. 3. Effects of exercise training on the collagen, elastin, macrophage, SMC, MMP-2, MMP-9 and TIMP-1 content of the atherosclerotic plaques and the calculated MMP-9/TIMP-1 ratio. ${ }^{*} \mathrm{p}<0.05$, exercice (EX) versus control (CO) groups; ${ }^{+} \mathrm{p}<0.05$, EX versus sedentary $(\mathrm{S})$ groups. NS $=$ Not significant. 


\section{Blood Concentrations of MMPs and TIMP-1}

The MMPs and TIMP-1 values are shown in table 1. At baseline, there were no differences among groups. At the end of the study, serum MMP-2 levels were not statistically different between all groups $(p>0.05)$. Serum TIMP-1 concentrations were increased in the exercise compared to control and sedentary groups throughout the study ( $\mathrm{p}<0.05$ ), while serum MMP-9 levels and MMP-9/TIMP-1 ratio were significantly lower in the exercise group compared to the other groups of both genders $(\mathrm{p}<0.01)$

\section{Discussion}

In the present study, we investigated the effects of exercise training on the atherosclerotic plaque size and composition, using a well-documented animal model of atherosclerosis. A significant regression of atherosclerosis after a treadmill training program, independent of body weight reduction or lipid profile modulation, was found. Importantly, these effects were accompanied by favorable alterations in plaque structure, such as an increase in collagen and elastin component, a decrease in macrophage number and a decrease in metalloproteinase activity. To our knowledge, this is the first study to demonstrate exercise-induced favorable effects on plaque composition and MMPs and TIMP-1 levels in advanced atherosclerotic lesions. Further, those effects of exercise training on plaque composition were documented in female animals as well. Of interest, changes in MMPs activity in the atherosclerotic plaques were parallel to those observed in the systemic circulation.

In the present study, exercise training had nonsignificant effects on total cholesterol, triglycerides and body weight compared to control and sedentary groups. In contrast, exercise studies in humans [11] and limited data from animals [12] have suggested that the anti-atherogenic mechanisms of exercise are at least partially related to lipid lowering and weight loss. A plausible explanation for this difference is related to the experimental model, since atherosclerosis in apoE-deficient mice is associated with a strong inflammatory and oxidative process $[13$, 14]. Thus, the exercise-induced regression of atherosclerosis in the animal model of the present study could be ascribed to other than traditional cardiovascular risk factors modification mechanisms, known as pleiotropic, affecting plaque burden and composition.

Exercise and Plaque Composition
Until now, little information has been available for possible gender-related differences in the effects of exercise, because most of the studies have focused on male mice [15]. The present study supports the notion that female apoE $\mathrm{E}^{-/-}$mice can be used as an animal model to study the pathophysiologic mechanisms of atherosclerosis and the influence of several interventions.

Vulnerable plaques are recognized by a number of histological characteristics, including decreased amount of collagen and elastin, low number of SMCs, large necrotic core, and excessive accumulation of macrophages and thinning of the fibrous cap [16]. It is widely accepted that cardiovascular events result mainly from the rupture of the vulnerable atherosclerotic plaque. The present study demonstrated that exercise training suppressed macrophage infiltration and that effect was associated with an increase in collagen and elastin content, especially at the fibrous cap area. On the other hand, the number of SMCs was slightly increased. The aforementioned exercise-induced changes in extracellular and cellular components of the atherosclerotic plaques are extremely important indicating beneficial alterations in atherosclerotic plaque composition. In the present study, switching from highfat to normal diet had negligible effects on atherosclerosis progression and plaque stabilization. Previous research has shown dietary cholesterol withdrawal to stabilize coronary atherosclerotic lesions in miniature pigs [17]. Although we used another animal model of atherosclerosis, we cannot rule out the possibility of a synergistic effect of normal diet plus exercise that exercise alone would not have produced.

Previous studies have reported a regression in atherosclerotic lesion size after exercise, but mostly the composition of atherosclerotic plaques and MMP activity was not assessed [3, 9, 18-21]. MMPs, as mediators of ECM degradation, are involved at several stages of the atherosclerotic process, such as inflammatory cell infiltration, vascular SMC migration, fibrous cap thinning and rupture of the atherosclerotic plaques [5]. On the other hand, TIMPs counterbalance MMP activity, and therefore the net proteolytic activity reflects the MMP/TIMP balance [4]. Growing evidence suggests that the absolute changes of individual MMPs and the MMP/TIMP ratio possess a key role in the atherosclerotic plaque vulnerability [22]. In animal studies, double knockout apoE ${ }^{-/-} \mathrm{MMP}_{-9^{-/-}}$ mice exhibited less severe and more stable atherosclerotic lesions after high-fat diet compared to $\mathrm{apoE}^{-/-}$mice [23]. Downregulated serum TIMP-1 levels have also been related to carotid plaque vulnerability [24]. In the present study, the concentrations of MMP-9 in the atheroscle- 


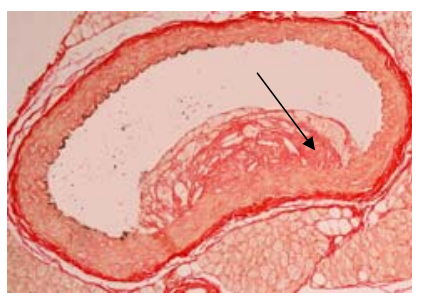

\section{COm (S-R)}

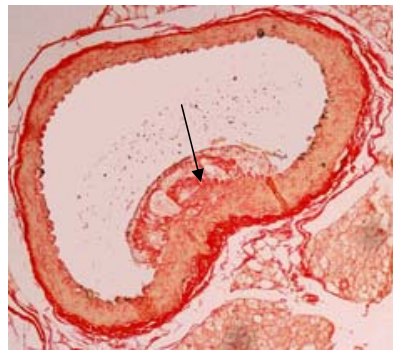

Sm (S-R)

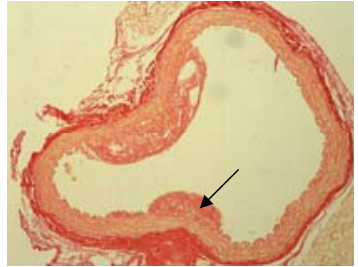

EXm (S-R)

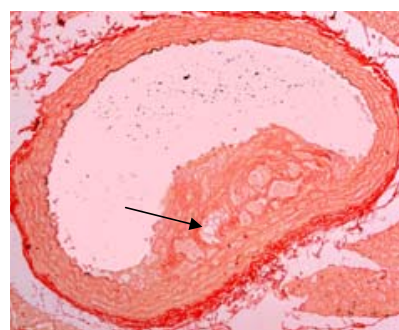

$\operatorname{COf}(\mathrm{S}-\mathrm{R})$

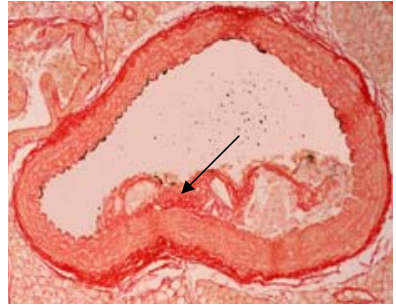

$\mathrm{Sf}(\mathrm{S}-\mathrm{R})$

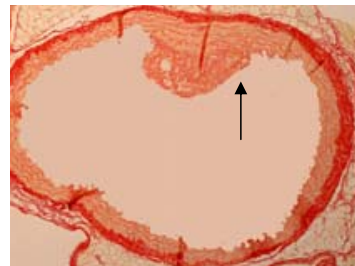

$\operatorname{EXf}(S-R)$

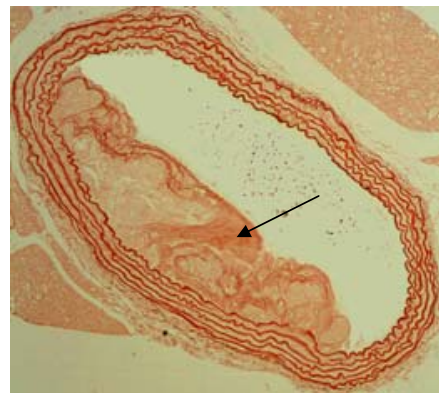

COm (Orcein)

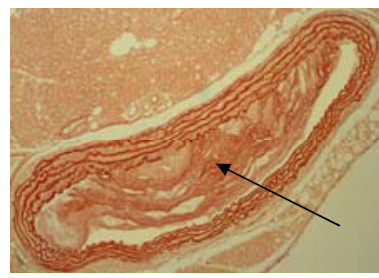

Sm (Orcein)

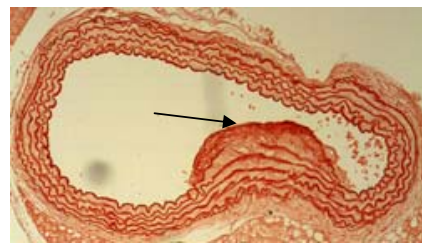

EXm (Orcein)
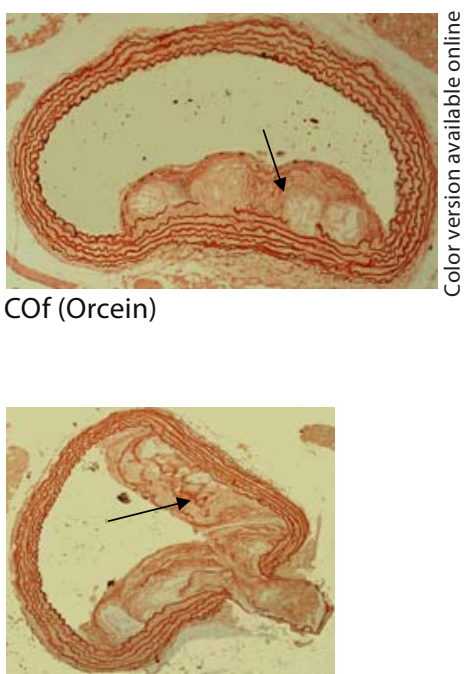

Sf (Orcein)

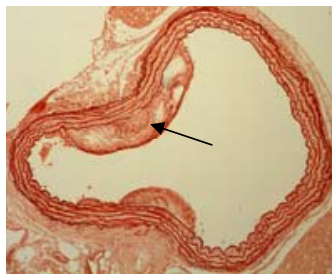

EXf (Orcein)

Fig. 4. Representative examples of the effects of exercise on the collagen (Sirius red, S-R) and elastin (Orcein) content of the atherosclerotic plaques. $\mathrm{COm}=\mathrm{Control}$ male; $\mathrm{COf}=$ control female; $\mathrm{Sm}=$ sedentary male; $\mathrm{Sf}=$ sedentary female; EXm = exercise male; EXf = exercise female. Arrows show positive stained areas.

Fig. 5. Representative sequential sections of the exercise and sedentary male apoE $\mathrm{E}^{-/-}$ mice groups showing the co-localization of macrophages and MMP-9. Atherosclerotic lesions staining with anti-Mac-3 antibody (macrophages) or anti-MMP-9. $\mathrm{EXm}=$ Exercise male; $\mathrm{Sm}=$ sedentary male. Arrows show positive stained areas.

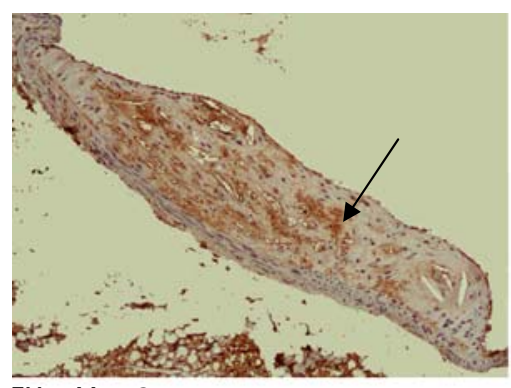

EXm Mac-3

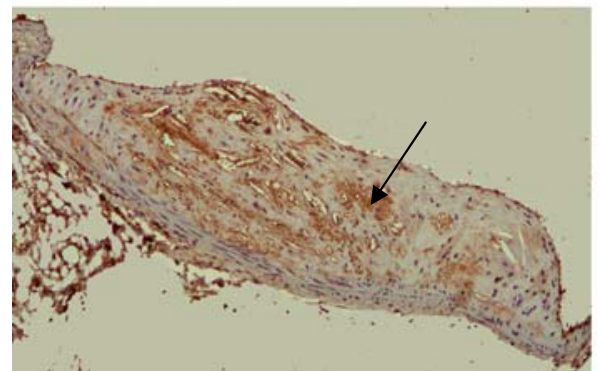

EXm MMP-9

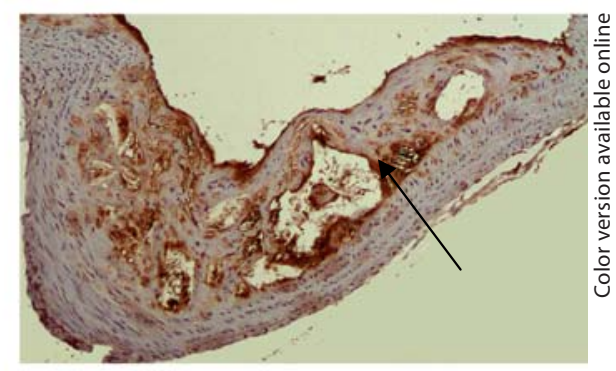

Sm Mac-3

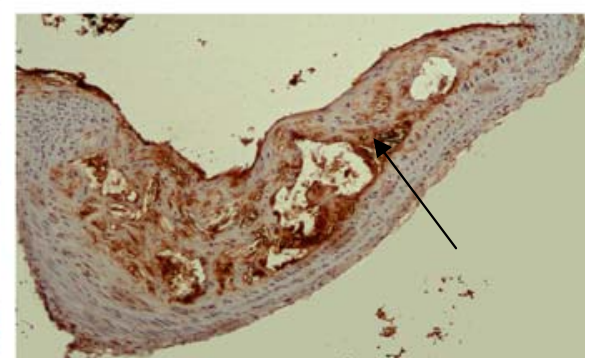

Sm MMP-9 
rotic plaques were reduced, while TIMP-1 levels were increased in the exercise animals. The MMP-9/TIMP-1 ratio showed a considerable reduction in the exercise compared to the untrained animals. These findings are of potential clinical importance, since human studies have demonstrated an association between MMP-9, atherosclerotic plaque vulnerability and subsequent cardiovascular events $[25,26]$.

The aforementioned effects of exercise training on MMPs and TIMP-1 may be related to the anti-inflammatory effects of exercise. In our study, exercise training suppressed the number of macrophages, which co-localized with the reduced MMP-9 area in the atherosclerotic plaques. Concerning macrophages and their derivatives, foam cells, as the predominant sources of MMP-9, this provides a plausible mechanism by which exercise modulated MMPs activity in our animal model. Furthermore, the reduction of pro-inflammatory and the increased expression of anti-inflammatory cytokines are well-known effects of exercise [1]. Pro-inflammatory (for example, TNF- $\alpha$ ) and anti-inflammatory (for example, IL-10) cytokines have been proved to trigger expression and activity of MMPs and TIMPs, respectively [27-29]. Perhaps lower numbers of macrophages produced smaller amounts of inflammatory cytokines and MMPs [30]. On the other hand, the number of SMCs and their product MMP-2 remained unaffected by exercise training. These findings could suggest MMP-2 expression as a prerequisite for SMC migration. Finally, NO inhibits MMP-2 and MMP-9 activity and augments TIMP activity [31]. Accordingly, the NO production induced by exercise might have halted atherosclerosis progression by a dual mechanism: attenuation of endothelial dysfunction and reduction of MMP activity [32]. It is suggested, therefore, that exercise training switched MMP/TIMP ratio to lower proteolytic activity by interacting with the inflammatory signaling pathways at several levels.

Exercise training significantly altered plasma MMP-9 and TIMP-1 levels in parallel with their concentrations in the atherosclerotic plaques. Besides this, unchanged MMP-2 content in the atherosclerotic plaques was mirrored in the plasma levels. Only few studies have thus far compared the MMP activity in the atherosclerotic plaque to that of the systemic circulation [33]. The findings from this study support the notion that those circulating biomarkers may be used to monitor inflammatory process in the plaque.

The principal limitation of this study was that advanced atherosclerotic plaques in mice rarely develop into rupture or erosion, the final evolution stage of atherosclerosis in humans. We must underline that the components of the atherosclerotic plaque (for example, macrophages and collagen) constitute surrogate markers of plaque vulnerability. Despite the observed beneficial changes in those cellular and acellular components, our study did not document direct effects on plaque rupture. Thus, the extrapolation of our results to humans should be done with caution. Nevertheless, lesions from apoE ${ }^{-/-}$ mice fed Western-type diet represent several histological characteristics of human atherosclerotic plaques, so their study facilitates the evaluation of atherosclerosis development and the effects of anti-atherogenic interventions on plaque composition. Finally, it is generally accepted that other than lipid-related mechanisms are mostly implicated in atherosclerosis induction in apoE knockout mice [34]. Although we did not measure all lipid fractions, the present and previous studies emphasize the role of inflammation and oxidative stress in animal atherosclerosis development, which is drastically different from human studies. Finally, switching to normal diet may have influenced our results, despite the absence of evident differences between control and sedentary groups.

In conclusion, the results of the present study demonstrated that exercise training prevented the progression of atherosclerosis and exerted favorable effects on atherosclerotic plaque composition. Modification of MMPs and TIMP-1 activity, macrophage concentration, collagen and elastin content of atherosclerotic plaque most likely accounted for the atheroprotective effects of exercise training in this experimental model.

\section{Acknowledgements}

This work was supported by the European Social Fund and National Resources - (EPEAEK II) 'HERAKLEITOS' and 'PYTHAGORAS I'. There was also financial support from the joint research and technology project 'Hellas-Slovenia', the Ministry for Development, General Secretariat for Research and Technology, the project 'KAPODISTRIAS' and the Hellas-Germany project 'IKYDA' from the Greek State Scholarships Foundation. N.P.E.K. was awarded a grant by the Alexander S. Onassis Public Benefit Foundation. 


\section{References}

$\checkmark 1$ Kadoglou NP, Iliadis F, Angelopoulou N, Perrea D, Ampatzidis G, Liapis CD, Alevizos $\mathrm{M}$ : The anti-inflammatory effects of exercise training in patients with type 2 diabetes mellitus. Eur J Cardiovasc Prev Rehabil 2007;14: 837-843.

-2 Nanni S, Melandri G, Hanemaaijer R, Cervi V, Tomasi L, Altimari A, Van Lent N, Tricoci P, Bacchi L, Branzi A: Matrix metalloproteinases in premature coronary atherosclerosis: influence of inhibitors, inflammation, and genetic polymorphisms. Transl Res 2007; 149:137-144.

-3 Okabe TA, Shimada K, Hattori M, Murayama T, Yokode M, Kita T, Kishimoto C: Swimming reduces the severity of atherosclerosis in apolipoprotein $\mathrm{E}$ deficient mice by antioxidant effects. Cardiovasc Res 2007;74:537545.

4 Kadoglou NP, Liapis CD: Matrix metalloproteinases: contribution to pathogenesis, diagnosis, surveillance and treatment of abdominal aortic aneurysms. Curr Med Res Opin 2004;20:419-432.

-5 Kadoglou NP, Daskalopoulou SS, Perrea D, Liapis CD: Matrix metalloproteinases and diabetic vascular complications. Angiology 2005;56:173-189.

-6 Mackey AL, Donnelly AE, Swanton A, Murray F, Turpeenniemi-Hujanen T: The effects of impact and non-impact exercise on circulating markers of collagen remodelling in humans. J Sports Sci 2006;24:843-848.

-7 Thorp E, Cui D, Schrijvers DM, Kuriakose G, Tabas I: Mertk receptor mutation reduces efferocytosis efficiency and promotes apoptotic cell accumulation and plaque necrosis in atherosclerotic lesions of Apoe ${ }^{-/-}$mice. Arterioscler Thromb Vasc Biol 2008;28:14211428.

-8 Naura AS, Hans CP, Zerfaoui M, Errami Y, Ju J, Kim H, Matrougui K, Kim JG, Boulares $\mathrm{AH}$ : High-fat diet induces lung remodeling in ApoE-deficient mice: an association with an increase in circulatory and lung inflammatory factors. Lab Invest 2009;89:124312451.

-9 Pynn M, Schäfer K, Konstantinides S, Halle $\mathrm{M}$ : Exercise training reduces neointimal growth and stabilizes vascular lesions developing after injury in apolipoprotein E-deficient mice. Circulation 2004;109:386-392.

- 10 Tangirala RK, Rubin EM, Palinski W: Quantitation of atherosclerosis in murine models: correlation between lesions in the aortic origin and in the entire aorta, and differences in the extent of lesions between sexes in LDL receptor-deficient and apolipoprotein E-deficient mice. J Lipid Res 1995;36:2320-2328.

11 Puglisi MJ, Fernandez ML: Modulation of C-reactive protein, tumor necrosis factor- $\alpha$, and adiponectin by diet, exercise, and weight loss. J Nutr 2008;138:2293-2296.
12 Edwards JM, Neeb ZP, Alloosh MA, Long X, Bratz IN, Peller CR, Byrd JP, Kumar S, Obukhov AG, Sturek M: Exercise training decreases store-operated $\mathrm{Ca}^{2+}$ entry associated with metabolic syndrome and coronary atherosclerosis. Cardiovasc Res 2010;85:631-640.

13 Thorngate FE, Rudel LL, Walzem RL, Williams DL: Low levels of extrahepatic nonmacrophage apoE inhibit atherosclerosis without correcting hypercholesterolemia in apoE-deficient mice. Arterioscler Thromb Vasc Biol 2000;20:1939-1945.

14 Miyata M, Smith JD: Apolipoprotein E allele-specific antioxidant activity and effects on cytotoxicity by oxidative insults and $\beta$ amyloid peptides. Nat Genet 1996;14:55-61.

15 Zenovich AG, Panoskaltsis-Mortari A, Caron GJ, Kolb AG, Fremming R, Nelson WD, Taylor DA: Sex-based differences in vascular repair with bone marrow cell therapy: relevance of regulatory and Th2-type cytokines. Transplant Proc 2008;40:641-643.

-16 Narula J, Garg P, Achenbach S, Motoyama S, Virmani R, Strauss HW: Arithmetic of vulnerable plaques for noninvasive imaging. Nat Clin Pract Cardiovasc Med 2008;5(suppl 2):S2-S10.

17 Verhamme P, Quarck R, Hao H, Knaapen M, Dymarkowski S, Bernar H, Van Cleemput J, Janssens S, Vermylen J, Gabbiani G, Kockx M, Holvoet P: Dietary cholesterol withdrawal reduces vascular inflammation and induces coronary plaque stabilization in miniature pigs. Cardiovasc Res 2002;56:135-144

18 Napoli C, Williams-Ignarro S, De Nigris F, Lerman LO, Rossi L, Guarino C, Mansueto G, Di Tuoro F, Pignalosa O, De Rosa G, Sica $\mathrm{V}$, Ignarro LJ: Long-term combined beneficial effects of physical training and metabolic treatment on atherosclerosis in hypercholesterolemic mice. Proc Natl Acad Sci USA 2004;101:8797-8802.

19 Ramachandran S, Penumetcha M, Merchant NK, Santanam N, Rong R, Parthasarathy S: Exercise reduces preexisting atherosclerotic lesions in LDL receptor knock out mice. Atherosclerosis 2005;178:33-38.

20 Shimada K, Kishimoto C, Okabe TA, Hattori M, Murayama T, Yokode M, Kita T: Exercise training reduces severity of atherosclerosis in apolipoprotein E knockout mice via nitric oxide. Circ J 2007;71:1147-1151.

21 Niebauer J, Maxwell AJ, Lin PS, Wang D, Tsao PS, Cooke JP: NOS inhibition accelerates atherogenesis: reversal by exercise. Am J Physiol Heart Circ Physiol 2003;285:H535H540.

22 Cheng M, Hashmi S, Mao X, Zeng QT: Relationships of adiponectin and matrix metalloproteinase- 9 to tissue inhibitor of metalloproteinase-1 ratio with coronary plaque morphology in patients with acute coronary syndrome. Can J Cardiol 2008;24:385-590.
23 Luttun A, Lutgens E, Manderveld A, Maris K, Collen D, Carmeliet P, Moons L: Loss of matrix metalloproteinase- 9 or matrix metalloproteinase-12 protects apolipoprotein Edeficient mice against atherosclerotic media destruction but differentially affects plaque growth. Circulation 2004;109:1408-1414.

24 Ding S, Zhang M, Zhao Y, Chen W, Yao G, Zhang C, Zhang P, Zhang Y: The role of carotid plaque vulnerability and inflammation in the pathogenesis of acute ischemic stroke. Am J Med Sci 2008:336:27-31.

25 Galis ZS, Sukhova GK, Lark MW, Libby P: Increased expression of matrix metalloproteinases and matrix degrading activity in vulnerable regions of human atherosclerotic plaques. J Clin Invest 1994;94:2493-2503.

26 Loftus IM, Naylor AR, Goodall S, Crowther M, Jones L, Bell PR, Thompson MM: Increased matrix metalloproteinase-9 activity in unstable carotid plaques. A potential role in acute plaque disruption. Stroke 2000;31: 40-47.

27 Becker JW, Marcy AI, Rokosz LL, Axel MG, Burbaum JJ, Fitzgerald PM, Cameron PM, Esser CK, Hagmann WK, Hermes JD, et al: Stromelysin-1: three-dimensional structure of the inhibited catalytic domain and of the C-truncated proenzyme. Protein Sci 1995;4: 1966-1976.

28 Nabata A, Kuroki M, Ueba H, Hashimoto S, Umemoto T, Wada H, Yasu T, Saito M, Momomura S, Kawakami M: C-reactive protein induces endothelial cell apoptosis and matrix metalloproteinase-9 production in human mononuclear cells: implications for the destabilization of atherosclerotic plaque. Atherosclerosis 2008; 196:129-135.

29 Lacraz S, Nicod LP, Chicheportiche R, Welgus HG, Dayer JM: IL-10 inhibits metalloproteinase and stimulates TIMP-1 production in human mononuclear phagocytes. J Clin Invest 1995;96:2304-2310.

30 DeGraba TJ: Immunogenetic susceptibility of atherosclerotic stroke: implications on current and future treatment of vascular inflammation. Stroke 2004;35(suppl 1):27122719.

31 Gurjar MV, Sharma RV, Bhalla RC: eNOS gene transfer inhibits smooth muscle cell migration and MMP-2 and MMP-9 activity. Arterioscler Thromb Vasc Biol 1999;19: 2871-2877.

32 Roberts CK, Chen AK, Barnard RJ: Effect of a short-term diet and exercise intervention in youth on atherosclerotic risk factors. Atherosclerosis 2007;191:98-106.

33 Turu MM, Krupinski J, Catena E, Rosell A, Montaner J, Rubio F, Alvarez-Sabin J, Cairols M, Badimon L: Intraplaque MMP-8 levels are increased in asymptomatic patients with carotid plaque progression on ultrasound. Atherosclerosis 2006;187:161-169. 